\title{
Coupled numerical-experimental study of an armour perforation by the armour-piercing projectiles
}

\author{
B. Zduniak, A. Morka \& T. Niezgoda \\ Department of Mechanics and Applied Computer Science, \\ Military University of Technology, Poland
}

\begin{abstract}
This paper concerns an analysis of target penetration by a selected armourpiercing (AP) projectile: 7.62x54R with steel core. Numerical and experimental research was carried out. The aim of this work was a comparison of the results obtained in real conditions of ballistic test and computer simulation. In this study, two three-dimensional targets and the core of the projectile were built. The structure of the projectile is complex, but steel core plays the main role in the perforation process. Then the numerical model of the projectile was reduced to describe only steel core dynamics. The 3D Element Free Galerkin method is applied to solve the problem under consideration. The algorithm implemented in the Ls-Dyna code was used. Space discretization of the analyzed problem was prepared by means of the HyperWorks Software (HyperMesh module). The total amount of the elements reaches 500000 in this model. The Johnson-Cook constitutive model is applied to describe the behaviour of the metallic parts: steel layers and the projectile's core. The experimental results were obtained using a high speed video-camera. The target penetrations by the projectile were recorded. The processing of the data obtained from a high speed camera was carried out by means of the TEMA Software. In this paper, a good correlation between the numerical and experimental results was obtained. A lot of interesting mechanical effects observed during the experiment were analyzed.
\end{abstract}

Keywords: penetration, perforation, numerical model, constitutive model, armour. 


\section{Introduction}

The paper describes a coupled numerical-experimental study of an armour perforation by a given type of projectile. An analysis of an AP $7.62 \times 54$ R type projectile with steel core was carried out. The structure of the projectile is complex, but steel core plays the main role in perforation process. The numerical model of projectile was reduced to describe only steel core dynamics. Two types of targets are used in modeling. The numerical simulations were performed using the Element Free Galerkin Method (EFG) implemented in LS-DYNA code. Three dimensional numerical models for each version of impact were developed. The initial stage of the problem is presented in Figure 1.

Time $=0$
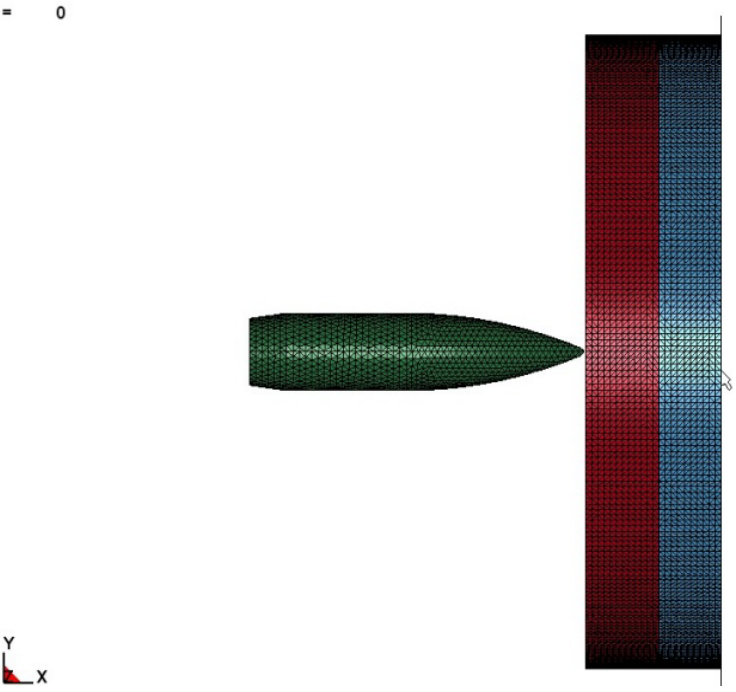

Figure 1: The initial stage of the problem. The target block with perpendicular impact of the 7.62 type projectile on target.

Three-dimensional models of the projectile and target were developed with a strain rate and temperature dependant on material constitutive relations. An impact of the $7.62 \times 54 \mathrm{R} \mathrm{mm}$ armour-piercing projectile on a $6 \mathrm{~mm}$ thick plate of steel and $5 \mathrm{~mm}$ thick plate of aluminum was modelled as the first type of target. The second type was made of four $5 \mathrm{~mm}$ thick aluminum plates. The target block with perpendicular impact of the 7.62 type projectile on target was assumed as reference. The perpendicular impacts are the most dangerous cases. The $7.62 \times 54 \mathrm{Rmm}$ projectile belongs to level III of the ballistic protection according to the STANAG 4569 standard. That type of projectile consists of a soft metal jacket, incendiary material and a hard steel core which is the crucial element for penetration effectiveness. The impact velocity equals $854 \mathrm{~m} / \mathrm{s}$. The geometry of this kind of projectile is shown in Figure 2. 


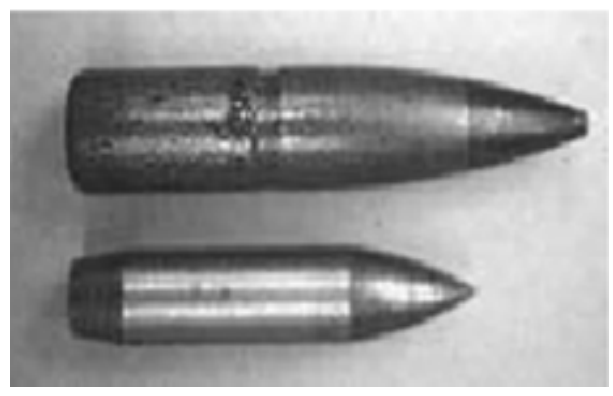

Figure 2: $\quad$ The geometry of projectile [1].

The experimental results were obtained by using a high speed video-camera. A scheme of the experiment is shown in Figure 3.

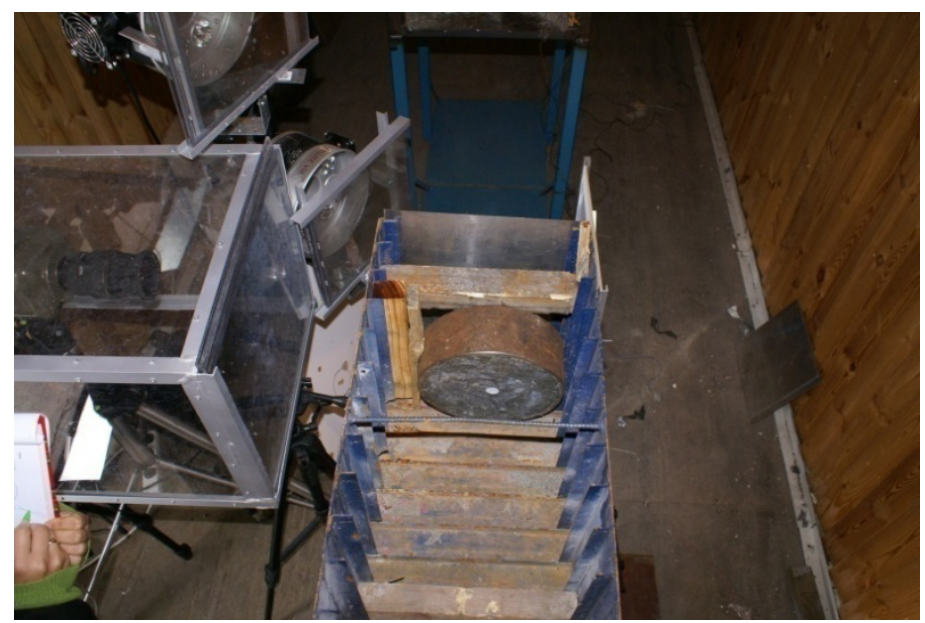

Figure 3: $\quad$ Scheme of the experimental test of impact.

The target penetration by projectile $7.62 \times 54 \mathrm{R}$ type AP was recorded. The processing of the data obtained from the high speed camera was carried out by means of the TEMA Software. The aim of this work was a comparison between the results obtained in real conditions of ballistic test and computer simulation.

\section{Numerical model}

For the purpose of this study numerical models of projectile and targets were constructed. Target can be built using three types of configurations: with steel plate and aluminum plate or four aluminum plates. Numerical models for panel's configurations: steel/aluminum plates, 4 aluminum plates are presented in Figure 4 (a), (b). 


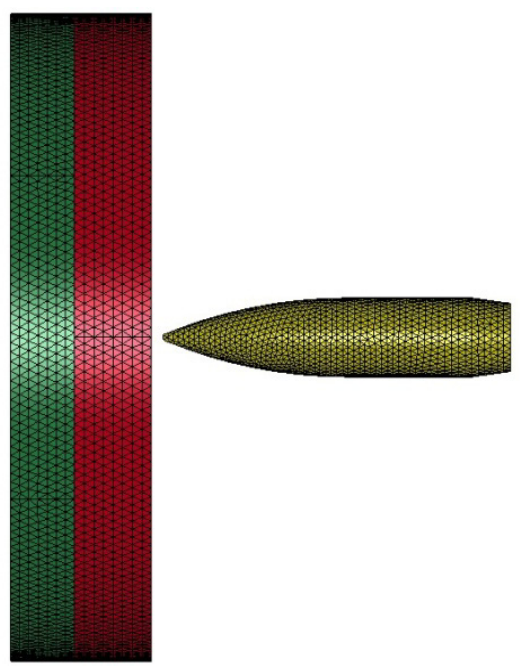

(a)

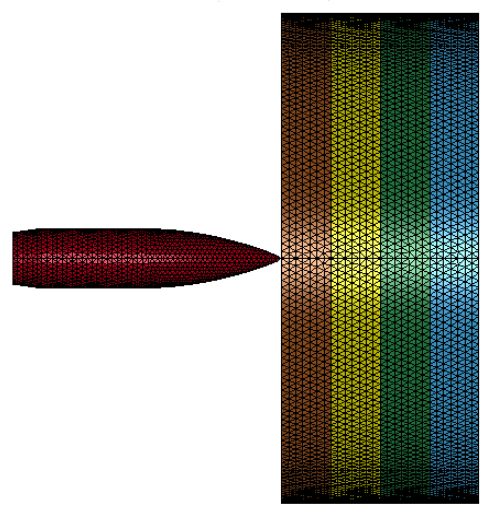

(b)

Figure 4: A 3D view of the mesh and geometry of the panel's configuration, (a) steel/ aluminum, (b) 4 aluminum plates.

Thickness of steel (ARMOX 500) plate equals 6 millimeters, thickness of aluminum (PA11) is 5 millimeters, diameters of all plates are 50 millimeters.

Figure 1 represents meshes of the numerical model consisting of three parts: the steel target backed by an aluminum plate and projectile's steel core. The typical node to node distance was equal to about $1 \mathrm{~mm}$ in all cases of targets and projectiles. The total number of elements per single case exceeded $500 \mathrm{k}$. The Johnson-Cook [2] constitutive model was chosen for armox500 and PA11. The data for constitutive model was derived from work [3]. The excessive deformations often observed in the perforation/penetration issues caused the choice of a meshless method as the method for the problem solution. The Element Free Galerkin (EFG) [4] method implemented in the LS-DYNA solver was selected $[5,6]$. EFG only uses a set of nodal points describing geometry of 
the body, no mesh in the classic sense is needed to define the problem. The initial condition was reduced to the given projectile velocity, $854 \mathrm{~m} / \mathrm{s}$. The boundary condition was assumed as the target plate fixed at its edge a 5 millimeters thick ring.

\section{Experimental setup}

This experimental work was carried out at a firing ground. The experimental setup is shown in Figure 3. There was a ballistic tunnel, in which firing takes place. A ballistic barrel was used for firing. 7.62x54R type AP of projectiles was used. All of projectiles were turned, because fuse of projectile induces a flash, what makes recording a film impossible. A turned projectile is shown in Figure 5 .

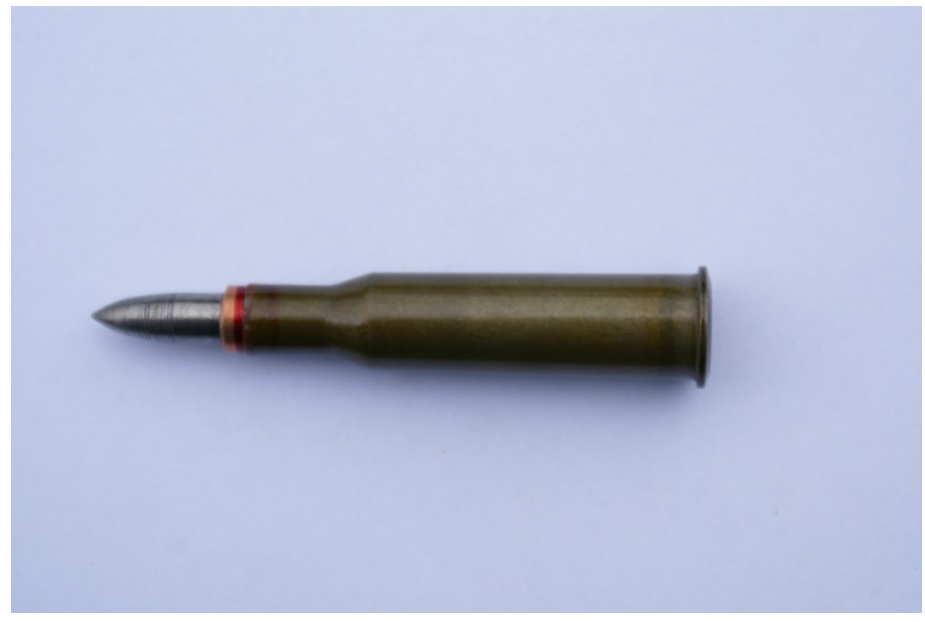

Figure 5: A turned projectile.

The target plate is fixed at its edge with a 5 millimeters thick ring. A high speed camera was put in the ballistic tunnel. It had a very resistant cover, made of a transparent polycarbonate.

\section{Results and discussion}

Computer simulations were performed for the selected cases. A high performance computing system based on the cluster architecture was used. This allowed us to assign 4 to $8 \mathrm{CPUs}$ per single job, limiting the total computing time to a reasonable level.

The first analysed variant was the steel-aluminum target. Armox500 type of steel was used and aluminum was of PA11 type.

This case underwent an entire perforation, in both numerical and experimental cases. It is presented below on a plot of kinetic energy, where ending kinetic energy value after target's perforation does not equal zero. 
Figure 6 shows the change in the kinetic energy of the projectile in time for the first case.

Figure 7 shows the change in the velocity of projectile in time.

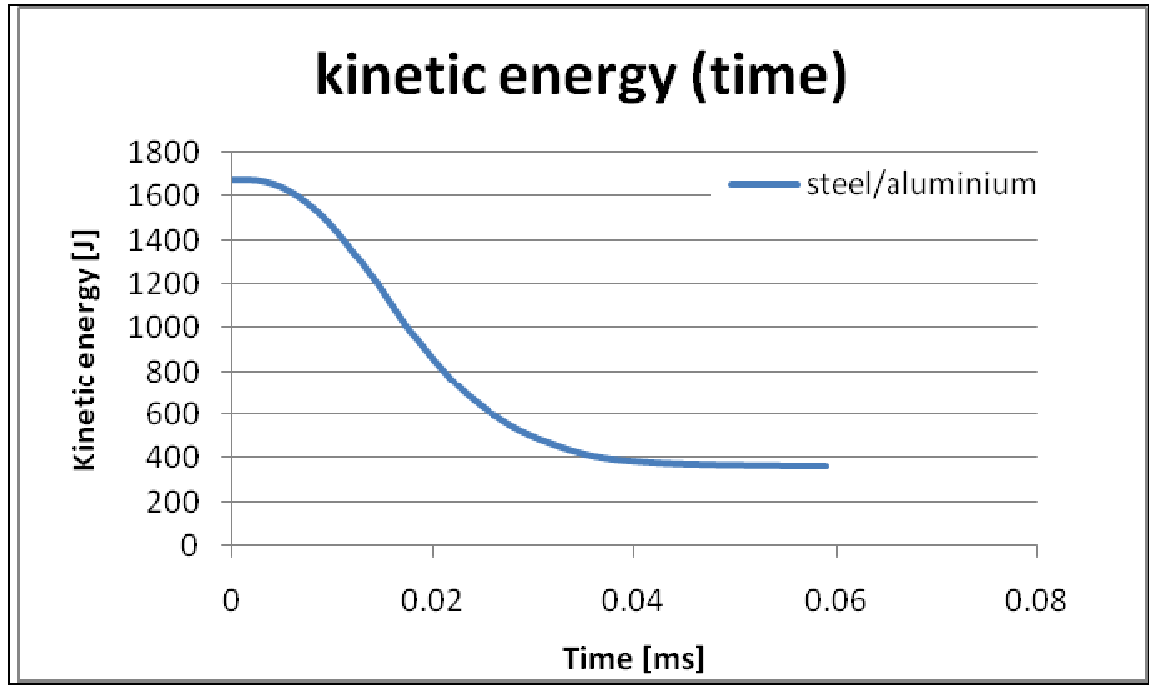

Figure 6: Change in the kinetic energy of projectile in time for steel/aluminum target for numerical simulation.

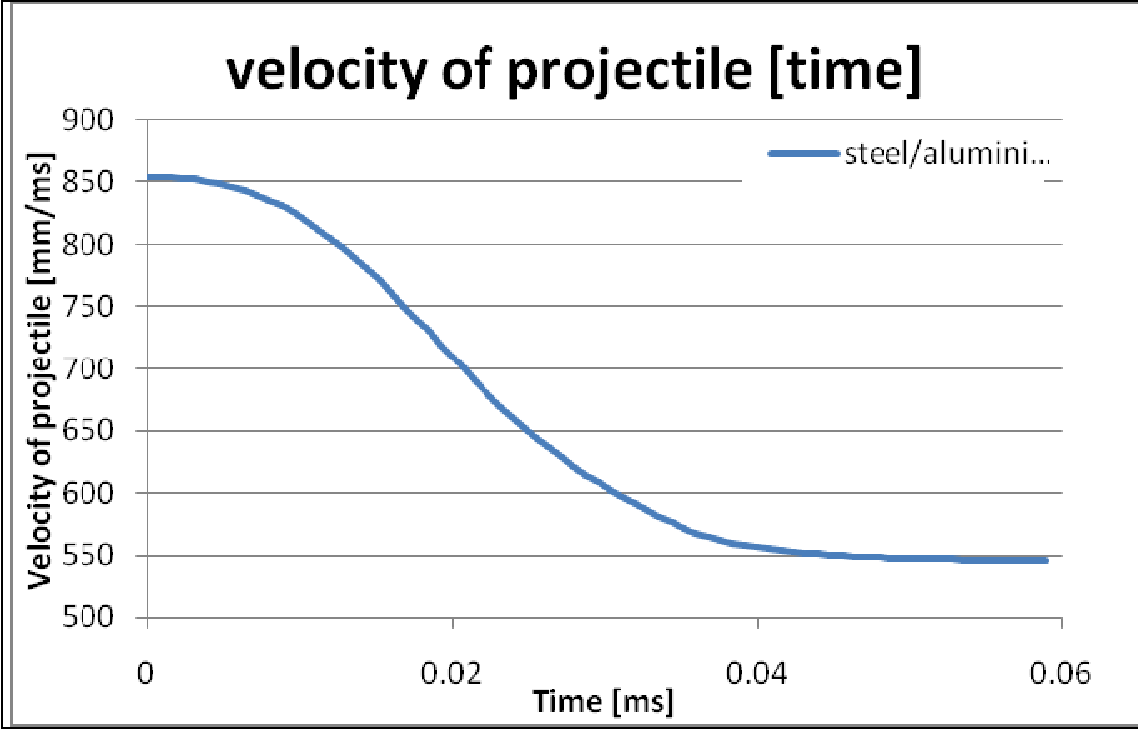

Figure 7: The change in the velocity of projectile in time (numerical result). 
The velocity of projectile in numerical model, after perforation of the target, equals about $550 \mathrm{~m} / \mathrm{s}$.

In experimental result, the velocity of the projectile after perforation of the target is about $576 \mathrm{~m} / \mathrm{s}$. This velocity is obtained from velocity's measurements on a short-circuit sensor. The velocity measurement obtained from a film analysis in Tema is impossible, because dust does not allow us to trace the projectile's path.

However, we can observe similar projectile's velocity values for both experimental and numerical cases.

Figure 8 presents a comparison of numerical model and experimental protective panel at the same time of a perforation process. This picture shows a very similar behavior of both targets. It means, that a damage model of numerical simulation is similar to fracture in real material, and that the used constitutive models and material parameters are compliant.

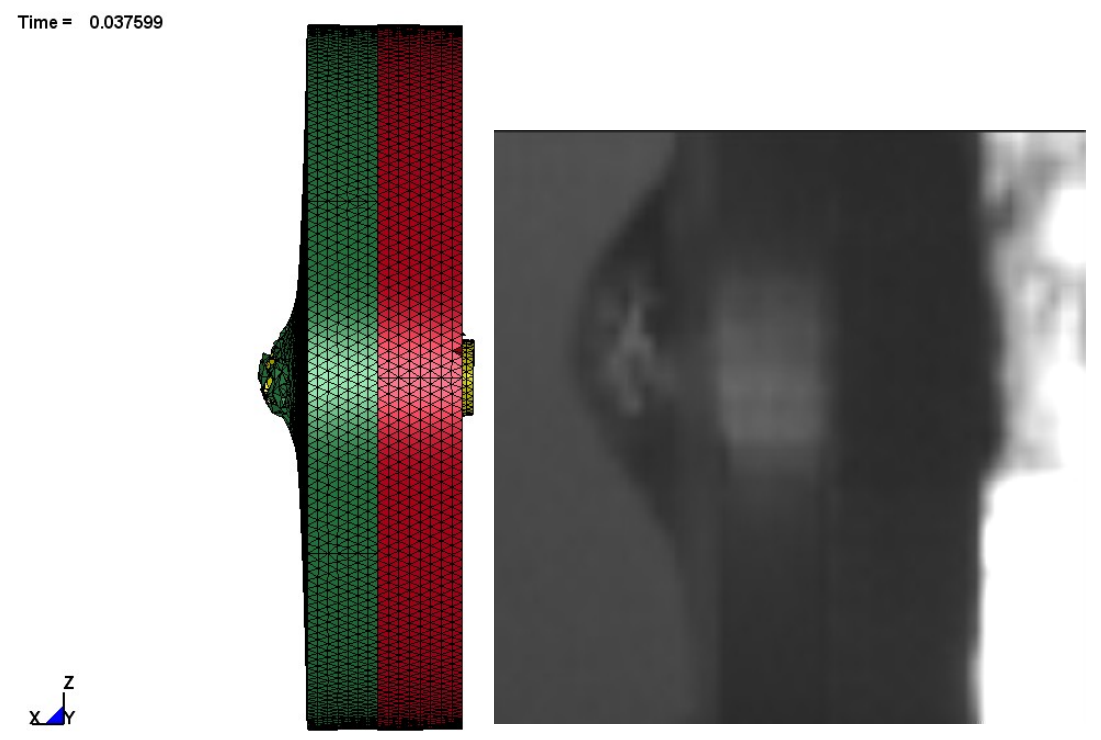

Figure 8: Comparison of numerical model and experimental protective panel at the same time of a perforation process for steel/aluminum target.

The second analysed variant is a target consisting of 4 aluminum plates. A type of aluminum was PA11.

This case underwent an entire perforation, in both numerical and experimental cases, which is shown in Figure 9. Figure 9 presents a change in the kinetic energy of the projectile in time for the second case for numerical model.

Figure 10 shows a change in the velocity of projectile in time. 


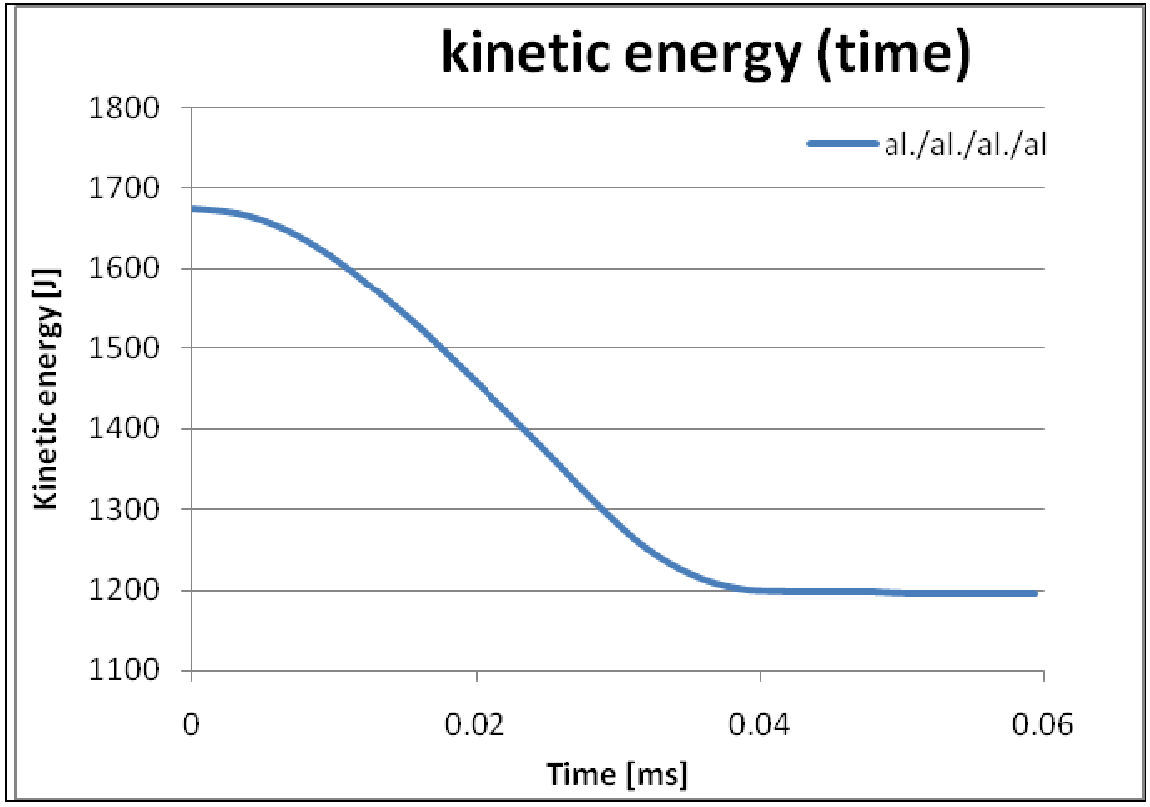

Figure 9: Change in the kinetic energy of the projectile in time for the second case for numerical model.

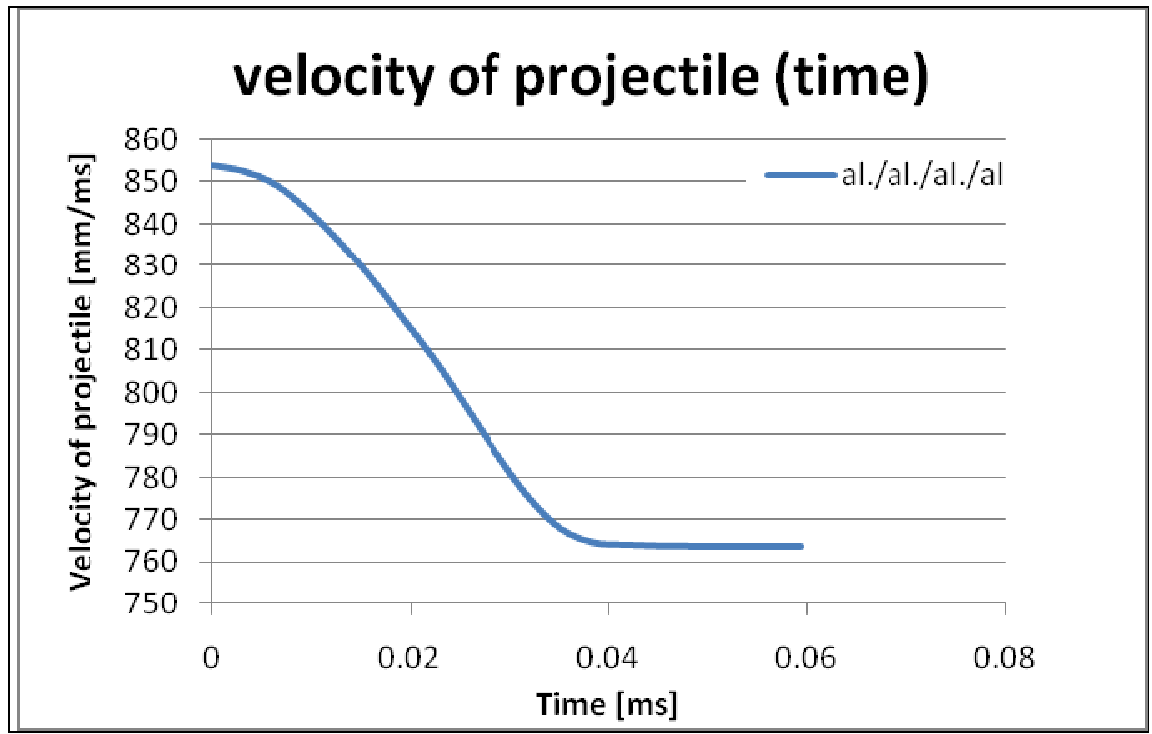

Figure 10: Change in velocity of projectile in time for second numerical model. 
The velocity of projectile in numerical model, after perforation of the target, equals about $762 \mathrm{~m} / \mathrm{s}$.

In experimental result, the velocity of the projectile after perforation of the target is about $706 \mathrm{~m} / \mathrm{s}$. This velocity is obtained from velocity's measurements on a short-circuit sensor. For this velocity value can have an influence on external factors, e.g. shock wave, fragments simulating projectile while using the short-circuit sensor. This may cause 7\% error between final experimental and numerical results.

A velocity obtained from Tema equals $709 \mathrm{~m} / \mathrm{s}$.

Figure 11 shows a comparison between the numerical model and experimental protective panel at the same time of a perforation process. This picture also shows a very similar behavior of both targets.

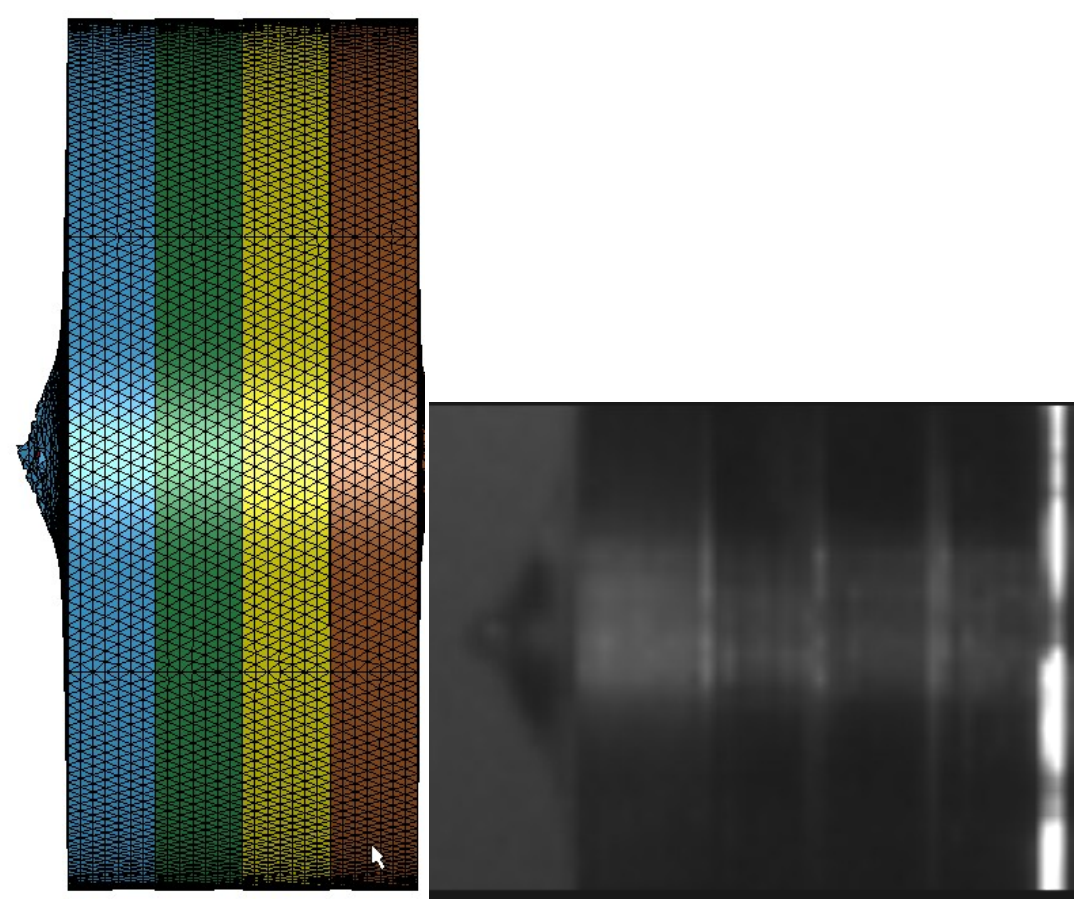

Figure 11: Comparison of the numerical model and experimental protective panel at the same time of a perforation process.

\section{Conclusions}

The presented analysis has provided only preliminary information about the comparison between the numerical and experimental model of the perforation problem. There is insufficient data about the mechanical properties of the steel, 
which the projectile is made of. This makes a quantitative assessment of the results difficult in many cases.

The most important task of this article is to show, that using numerical models allows us to reduce the cost of experimental research, which is very expensive. The correlation obtained in this job is enough to know if this target gives in perforation or not. A similar behaviour of penetration targets is also achieved. Plate materials were destroyed in similar ways. In this paper, a good correlation of the numerical and experimental results is received. Further work for finding a good damage parameters for steel projectile will be carried out.

\section{References}

[1] Paul J. Hogg, "Composites for ballistic applications", Department of Materials Queen Mary, University of London.

[2] G. R. Johnson, W. H. Cook, A constitutive model and data for metals subjected to large strains, high strain rates and high temperatures, Engineering Fracture Mechanics, Volume 21, Issue 1, p. 31-48, 1985.

[3] A. Morka, B. Jackowska, T. Niezgoda, Numerical study of the shape effect In the ceramic based ballistic panels, Journal of Kones Powertrain and Transport, Vol.16, No.4, 2009.

[4] C. Kambur, Assessment of Mesh-free Methods in LS-DYNA: Modeling of Barriers in Crash Simulation, 2004.

[5] J. O. Hallquist, LS-DYNA Theory Manual, Livermore Software Technology Corporation, 2006.

[6] LS-DYNA Keyword User's Manual, version 971, Livermore Software Technology Corporation, 2007. 